\title{
Detection of events in seismic time series by time-frequency methods
}

\author{
S. Gabarda and G. Cristóbal \\ Instituto de Optica (CSIC) \\ Serrano 121, 28006 Madrid, Spain \\ phone:+(34)915616800,fax: + (34)915645557,email: \{salvador,gabriel\}@optica.csic.es
}

\begin{abstract}
The detection of events in seismic time series has been a subject of great interest during the last thirty years. Most of the work in this area has been based on detecting special patterns or clusters in seismic data. In this paper, we present a new event detection method based on a time-frequency analysis through the Wigner Distribution (WD). Our method consists on defining an appropriate entropic measure through a suitable time-frequency distribution, acting as probability distribution function (PDF). We know from previous studies in the field that the information entailed by time-frequency representations (TFR) of time signals can be explored by means of different Rényi entropy measures. The non-positivity character of the WD implies that the classical Shannon entropy cannot be used and therefore it has been replaced by a generalized measure such as the Rényi entropy. However, due to the existence of multiple TFR normalizations, the so-called quantum normalization has been empirically selected here for this particular application. Our method is based on the identification of the events as those temporal clusters having the highest amount of information (entropy). The method is described and applied to different earthquake signals and volcanic tremors, using both real and synthetic data. Results are compared to other existing event detection methods.
\end{abstract}

Keywords: Seismic signals, Rényi entropy, pseudo-Wigner distribution.

\section{INTRODUCTION}

Feature extraction from seismic traces (time series) is an area of increasing interest since the 80s. This included the work of Hagen [1] who used the principal components of the correlation coefficients in the search for clusters of significant events; Bois [2] fitted autoregressive models to seismic trace segments; Dumay [3] used 'classical' features such as energy or zero crossings as discriminant variables; Lendzionowski [4] showed that certain instantaneous attributes allow instant identification of gaswater contact; Walden [5] proposed the use of energy traces, as well as temporal and frequency parameters, as discriminant attributes; Grubb [6] proposed the use of wavelet coefficients for both the identification and characterization of clusters in the traces. Other techniques have also been proposed based on the amplitude of the seismic signal and the variation of the deformation [7], the spectrogram (windowed Fourier transform) or by more complex methods such as those based on maximum entropy [8]. 
It is worthy to be noted that studies on the application of time-frequency analysis techniques, and diverse entropy measures, have been carried out for other type of signals such as for example, biomedical signals $[9,10]$. Dealing with time-frequency analysis, the Rényi entropy appears as one outstanding measure for determining the information content of a signal and it has deserved a great amount of interest in the literature [11-19]. However, the Rényi entropy can be approached under different normalization scenarios, having not a unique definition. This paper proposes one specific instant-wise normalization, as the most convenient among many others possibilities, for the analysis of discrete-time series. The aim of this method is to accurately detect significant events in seismic signals, but the results can be generalized and extended to other signals or applications.

The information content entailed by the TFRs of time series can be explored by means of different Rényi entropy measures. The Wigner distribution (WD) [20] constitutes a suitable TFR for non-stationary signal analysis . Due to its non-positivity property, the WD of a signal cannot be interpreted properly as a PDF. Therefore, a complexity measure based on the classical Shannon entropy cannot be used and a generalized measure such as the Rényi entropy is required. A generalized Rényi entropy measurement can provide a quantitative criterion to evaluate the importance of the information at a given time $t$, and it can be used for adaptively and automatically selecting parameters in a time-frequency analysis [11]. In this paper, we describe a new method of feature extraction in time series that could be of interest in geophysics for detecting clusters in seismic traces. Likewise, it is worth to mention that this method has also been tested with success by the authors for discriminating between normal and abnormal events in biomedical recordings such as phonocardiograms [21]. This paper is structured as follows. The mathematical background is outlined in section 2. The method is described in section 3. Section 4 presents the experimental results obtained testing the method with seismic and synthetic signals. Conclusions and future research work is outlined in section 5.

\section{THEORETICAL BACKGROUND}

\subsection{The pseudo-Wigner distribution}

This method deals with discrete-time signals. In this paper, discrete-time signals are considered as the result of a conversion from a given continuous signal $z$ captured by a seismic sensor by uniform sampling. Namely, $z[n]=z_{c}(n T)$. Here $z_{c}$ is the continuous signal, $T$ is the sampling period and $n$ is an integer number defined in the interval $0<n<\infty$. Also, square brackets have been used to indicate the discrete character of $z$ in contrast with the continuous character of $z_{c}$ indicated by round brackets, as a conventional notation.

The aim of this paper is to introduce a new event detection method for seismic traces. An event can be defined as a feature or cluster of interest that happens at a given time $t$. In a digital signal scenario, only the samples containing extraordinary events will be referred as events and the remaining samples will be considered as no-event containers. The key subject is then the use of the word extraordinary for defining an 
event. This term must be defined according to the kind of signal analyzed and the interest of the observer. For example, if the signal is a heartbeating, then the heart strokes are the events. In such an example these events can be characterized and compared to a pattern to decide if they are normal or not to diagnose a possible disease. The problem resides now in designing a method to detect and measure events in the most convenient way for the observer purposes. Focusing on seismic signals, we can consider two levels of detection. In a first scenario, seismographs can register signals that are not seismic, such as nuclear explosions for example. Hence, a first detection problem consists in identifying truly seismic signals. The second scenario appears once the signal has been accepted as seismic and some special observations in it can be interpreted as minor events and classified as a particular subclass, according to a given rule of classification, based on a predefined model. According to this, the different methods of analysis can be classified as supervised or unsupervised upon some a priori information that identify signal's performance as a recognizable event or, on the other side, for determining pattern of interest in the input data for modeling event representation [22].

This paper proposes an unsupervised measure based on the local entropy of the signal.. This means that 'a priori' knowledge of the events is not needed. Then, the output of the method can be interpreted as a probability value assigned to each sample of the series for detecting regions with 'special' activity. The classification of such events is out of the scope of this paper.

Formally, signals can be analyzed in the time domain, in the frequency domain and even in time-frequency domain. The last one presents many special characteristics justifying the attention received for a long time by the signal processing community. Time-frequency representations can be analyzed using a common analytical framework introduced by Cohen [23]. The TFR of a signal can be introduced by diverse existing definitions [24]. In this application, the Wigner distribution has been selected due to its excellent properties and because it can be regarded as a 'masterform' distribution from which the other existing representation can be derived [24]. The WD can be approximated for discrete signals by a formalism such as Eq. (1) due to Claasen and Mecklembräuker [25] or alternatively using Brenner's expression [26]. These approximations have been referred as pseudo-Wigner distributions (PWD) to take account that they do not exactly match the properties of the continuouos version.

$$
W[n, k]=2 \sum_{m=-\frac{N}{2}}^{\frac{N}{2}-1} z[n+m] *[n-m] e^{-i 2 \pi k\left(\frac{2 m}{N}\right)}
$$

This expression produces a vector whose elements contain information of the strength of the discrete frequencies existing in such temporal position $t=n$, and for a neighborhood of $n$. Eq. (1) can be interpreted as the discrete Fourier transform (DFT) of the product $r[n, m]=z[n+m] z *[n-m]$. Here $z^{*}$ indicates the complex-conjugate of signal $z$. This equation is limited to a spatial interval [-N/2,N/2-1]. In Eq. (1), $n$ and $k$ 
represent the time and frequency discrete variables respectively, and $m$ is a shifting parameter, which is also discrete. $W[n, k]$ is a matrix where every row is a vector representing the instantwise PWD for a given $n$. By scanning the temporal signal with a 1-D window of $N$ data, i.e., by shifting the window to all possible positions along the signal, the full instantwise PWD of the signal is produced.

Figure 1 shows an example of the graphical representation of the PWD at a given instant $n$ of a temporal signal. The PWD will present different magnitude coefficients at every position $t=n$ due to the data value changes along the time signal. A way to measure such differences in the PWD is by defining an instantwise measure, as it will be described in the following sections.

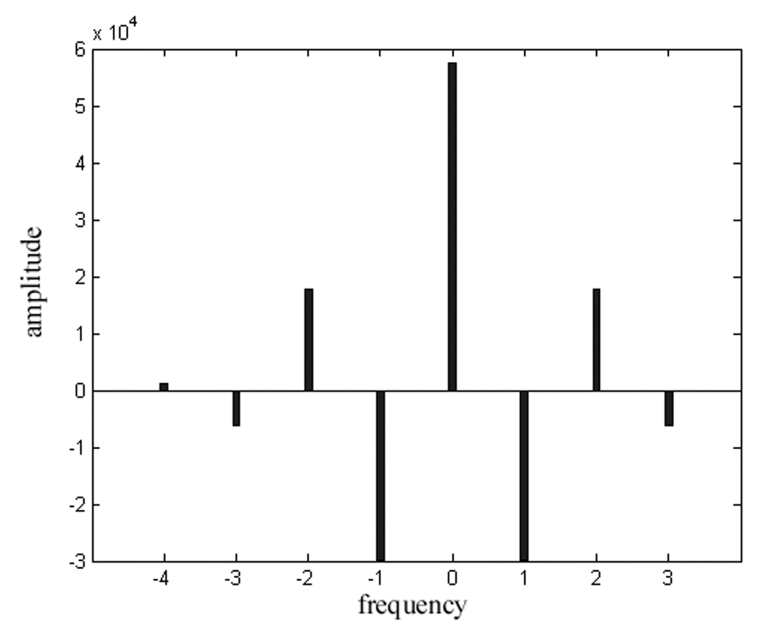

FIGURE 1. PWD of a given signal at time $t=n$

\subsection{A local Rényi entropy measure}

Once the local frequency content has been obtained by means of Eq. (1), a new entropy measure is required for extracting the information contained in a given position $n$. One of the possibilities is to use the Rényi entropy, which is a generalisation of the Shannon entropy, as a measure of the uncertainty or randomness of a signal, i.e., it is a measure of the information content of the signal.

The concept of entropy was initially proposed by Shannon [12] as a measure of the information content per symbol, coming from a stochastic information source. Later, Rényi [13] extended this notion to yield the generalized entropy. The measures of entropy based on the formulation due to Rényi and extracted from time-frequency distributions have been developed by different authors [14-17], with a significant contribution of P. Flandrin et al. [18] establishing the properties of such measures. The use of entropic measures extracted from positive time-frequency representations was done by Pitton et al. [19].

The Rényi entropy measure applied to a discrete time-frequency distribution $P[n, k]$ has the form

$$
R_{\alpha}=\frac{1}{1-\alpha} \log _{2}\left(\sum_{n} \sum_{k} P^{\alpha}[n, k]\right)
$$


Here $n$ is again the temporal discrete variable and $k$ the frequency discrete variable. $\alpha \geq 2$ are recommended values for time-frequency distribution measures [18]. Although the Rényi measures of time-frequency distributions formally resemble the original entropies, they do not have the same properties, conclusions and results derived from classical Information Theory. The positivity, $P[n, k] \geq 0$ will not be always preserved, along with the unity energy condition, $\sum_{n} \sum_{k} P[n, k]=1$. For fulfilling the later, some kind of normalization needs to be considered [14]. The normalization can be performed in various ways, leading to a variety of possible definitions $[11,18]$. Hence, the PWD as given by Eq. (1) must be normalized as a previous step to the Rényi entropy calculation.

Quantum Mechanics [27] inspires a normalization by assimilating the PWD of a given instant $t=n$ with a wave function and deriving its PDF by means of

$$
P[n, k]=\frac{W[n, k] W^{*}[n, k]}{\sum_{k}\left(W[n, k] W^{*}[n, k]\right)}=\frac{W^{2}[n, k]}{\sum_{k} W^{2}[n, k]}
$$

Squaring of $W$ is a consequence of the real character of the PWD, and the normalizing stage $i s$ affecting exclusively to index $k$, when the operation is restricted to a single position $n$ to satisfy the condition $\sum_{k} P[n, k]=1$ in such position.

Then, the general case in Eq. (2) for $\alpha=3$, gives

$$
R_{3}=-\frac{1}{2} \log _{2}\left(\sum_{n} \sum_{k} P^{3}[n, k]\right)
$$

This measure can be rewritten for a given $n$ as follows:

$$
R_{3}[n]=-\frac{1}{2} \log _{2}\left(\sum_{k} P^{3}[n, k]\right)
$$

Empirically, the 'quantum normalization' has shown to be most suitable for this application, and it was effectively used in the experimental examples later described. The values of $R_{3}[n]$ depend upon the size $N$ of the window used in Eq. (1) and it can be shown that they are within the interval $0 \leq R_{3}[n] \leq \log _{2} N$. Hence, the measure can be normalized by applying $\hat{R}_{3}[n]=R_{3}[n] / \log _{2} N$ in Eq. (5).

\subsection{Signal normalization and bias}

Due to the normalization applied in Eq. (3), $W[n, k]$ can be interpreted as a probability distribution. Moreover, it can be easily shown that the instant-wise entropy calculated by Eq. (5) is independent of the signal's amplitude $z[n]$. That is, $z[n]$ and $a \times z[n]$, where $a$ represents a real-positive constant, have both the same entropy. When the signal amplitude is zero-mean, the measure may be masked by the noise, if both, signal and noise, have zero-mean. To avoid this, a constant bias may be added to the signal, i.e., $z[n]+b$, with $b$ a real-positive constant. By setting $b>\| v[n] \mid$, where $v$ is the noise, such measure will be amplitude dependent and therefore it will be 
independent of the noise. This will be illustrated by the examples presented in Section 4.

\section{DESCRIPTION OF THE METHOD}

The aim of the proposed method is to provide valuable information about the significance of discrete-time signals events, by revealing their position and their spectral importance, starting from an unprocessed signal. This method facilitates the detection of events of interest, given the fact that only the amplitude of the signal is frequently not enough for providing a precisely information about the position and significance of the events.

In any case, the discrete-time signal to be treated requires a pre-processing step, basically consisting in keeping the values in a limited range and applying some bias to the signal in order to set all values above zero. Downsampling the signal is also possible for reducing the computational time. Once the discrete-time signal has been digitized and biased, the PWD of the data is obtained using Eq. (1) by means of a sliding window of $N$ values. In practice, $N$ will be a small even number (e.g.: $N=8$, 10 , etc.) to ascertain temporal localization of events. Anyhow, the exact value of this parameter will depend on the particular characteristics of the signal and the sampling frequency used to record it.

After calculating the PWD of the temporal signal, every temporal position $n$ has an associated vector of $\mathrm{N}$-components, representing its instantaneous PWD. Then, the Rényi entropy can be measured in an instantaneous way, by means of Eq. (5).

As a result of the previous procedure, a new temporal sequence of data is obtained, where normal and anomalous events can be better detected and visualized, by providing a more discriminative distinction between them. It is important to remark that parameters such as the window size or the bias value selected will produce changes in the shape and in the absolute value or the resulting entropy. The observed results have a meaning when comparing different signals submitted to identical processes. Finally, it is important to highlight that the quantum normalization is the cornerstone of the discriminative ability of this method.

\section{EXPERIMENTAL RESULTS}

In this section, some examples are presented for illustrating how the above described method works in the case of seismic signals. The first set of examples corresponds to some representative time series of such signals. Fig. 2a shows a plot from the October 17, 1989 Loma Prieta earthquake in the Santa Cruz Mountains, CA. Fig. $2 \mathrm{~b}$ shows the results after applying the current method. From this plot one can better track the presence of significant events. The next example in this area is presented in Fig. 3. Fig. 3a shows a prototypical volcanic tremor of the Timanfaya volcano (Canary Islands, Spain). Fig. 3b shows how this method is able to discriminate the low amplitude events from the high amplitude ones. 


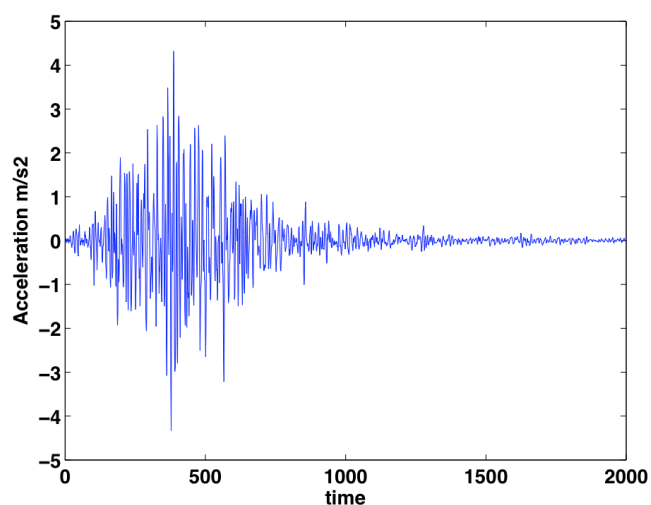

(a)

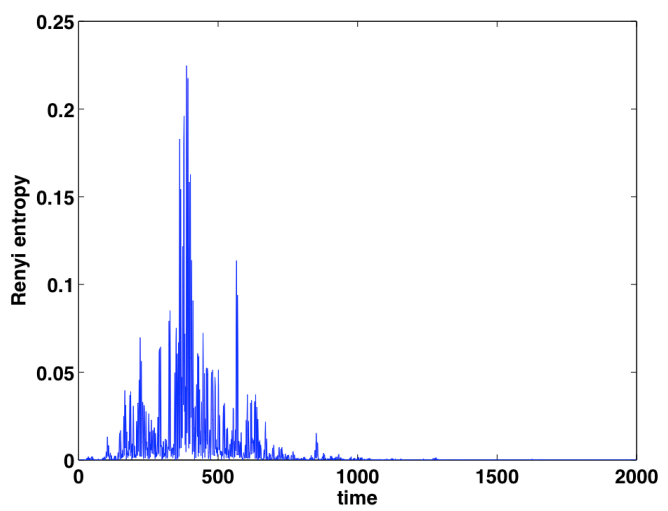

(b)

FIGURE 2. (a) Time history of the acceleration of the north-south component of the Santa Cruz record from the $200 \mathrm{~Hz}$ Loma Prieta earthquake in 1989. This record represents approximately twice the amplitude of the incident wave. (b) Events detected by the method described in the text. The PWD has been calculated using a window of $N=8$ data. Seismic data are courtesy of Joel Yellin at the Charles F. Richter Seismological Laboratory.

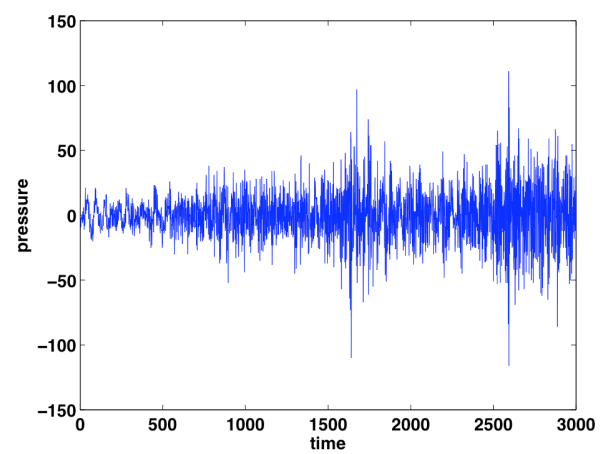

(a)

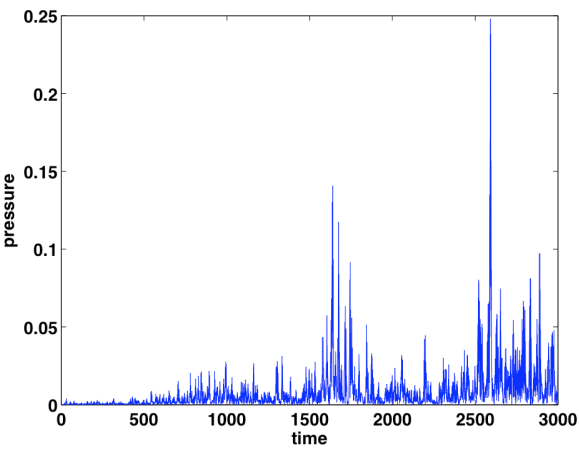

(b)

FIGURE 3. (a) Example of a volcanic tremor time series of the Timanfaya volcano (Canary Islands, Spain). b) Events detected by the described method. The PWD has been calculated using a window of $N=8$ data. Tremor data are courtesy of Alicia Garcia and Ramon Ortiz, Depto. Volcanologia, Museo Nacional de Ciencias Naturales (CSIC), Madrid, Spain.

The next example corresponds to a synthetic seismological signal. The mathematical model used to generate such signals is based on the method proposed by Axel Plešinger and Petr Kolár [28] and described herein. Seismic waves propagating through the interior of the Earth, so-called body waves, are described by the convolution of the source function (body-wave signal radiated by the earthquake) with the transfer function of the medium through which the source signal propagated to the point of observation. Both functions, especially the latter one, called Green's function, are complex functions of many variables. A convenient source function, allowing modeling of the rise time, the predominant frequency and the spectral selectivity (damping) of the source wavelet of a seismic body wave, is the so-called Berlage function: 


$$
w(t)=A t^{\alpha} e^{-\beta t} \sin (2 \pi f t)
$$

The required amplitude, rise time, damping and predominant frequency of the wavelet is modelled by proper selection of the constants $\mathrm{A}, \alpha, \beta$ and $f$, respectively. More complex wavelets are possible by superimposing two or more different and mutually arbitrarily delayed Berlage signals. The effect of the propagation path is simulated by the convolution of the source signal with a Green function in the form of an impulse trace, $i(t)$, consisting of an optional number of delta functions, $\delta\left(t_{k}\right)=1$, for $t_{k}=0$ and $\delta\left(t_{k}\right)=0$, for $t_{k} \neq 0$, each defined by its amplitude, polarity, and time delay,

$$
i(t)=\sum_{k=1}^{K} C_{k} \delta\left(t_{k}\right), \quad t_{k}=t-\tau_{k}
$$

This trivial Green function corresponds to the effect of simple reflections of the seismic wavelet on discontinuities at different depths. A further option is the superposition of random noise, $n(t)$, to the resulting convolved signal. Optional parameters are the signal-to-noise ratio and the number of samples over which the random noise is averaged in order to limit its high-frequency spectral components. Therefore, the final simulated signal is defined as

$$
y(t)=w(t) * i(t)+n(t)
$$

where $n(t)$ is the random noise.

In the following example, a simulated seismic function has been generated using Eq. (6), adding $2 \%$ of Gaussian noise to the signal. The resulting pulse train is shown in Fig. 4a. Such test wave has been subdued to two different filtering processes based on two different methods, one of them is the Rényi method described in this paper. The resulting filtered signal is shown in Fig. $4 \mathrm{~b}$.

The other method is based on Fisher statistics, widely used in the detection of infrasound events [29]. The Fisher ratio

$$
F=\frac{T(N-1) \sum_{t=1}^{T}\left[\sum_{n=1}^{N} x_{n t}\right]^{2}-\frac{1}{T}\left[\sum_{t=1}^{T} \sum_{n=1}^{N} x_{n t}\right]^{2}}{N(T-1) \sum_{t=1}^{T} \sum_{n=1}^{N} x_{n t}^{2}-\frac{1}{N} \sum_{t=1}^{T}\left(\sum_{n=1}^{N} x_{n t}\right)^{2}}
$$

indicates the correlation between a group of seismic signals, coming from several stations (coherence measure) [30]. In Eq. (9), $F$ is the Fisher ratio, $T$ is the timewindow over which the correlation is calculated, $N$ the number of stations and $x_{n t}$ the signal belonging to station $n$ at time $t$. In this example, Eq. (9) has been adapted to measure the coherence between two signals, one of them is the test signal shown in Fig. $4 \mathrm{a}$ and the other one is a pattern consisting in a sinusoidal wave, composed by 
100 samples of the same frequecy $f$ as that of the test signal, shifted over the test signal for instantwise calculation purposes. The result of this filtering process is shown in Fig. 4c.

The Fisher rate has been re-calculated changing the reference sinusoidal pattern to 50 $\mathrm{Hz}$ instead of the original $10 \mathrm{~Hz}$ pattern. The new outcome does not discriminate the signal from the noise as shown in Fig. 4d.

The comparison of results on Fig. 4 indicates that the Rényi method presents a high signal to noise discrimination, in an unsupervised way, presenting a sharp indication of the fundamental position of the pulse, while the Fisher ratio results in a rough indication of the pulse position, exclusively when the signals have a coincidence in their respective frequency content. If the frequency of the reference pattern is shifted away of the test signal frequency, the signal remains undetected. This is anyhow an expected result, provided that the two signals are now uncorrelated.

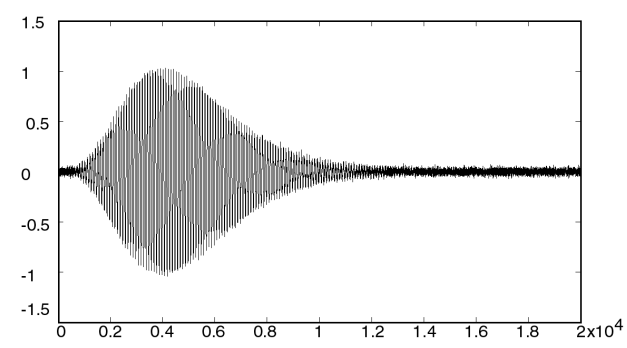

(a)

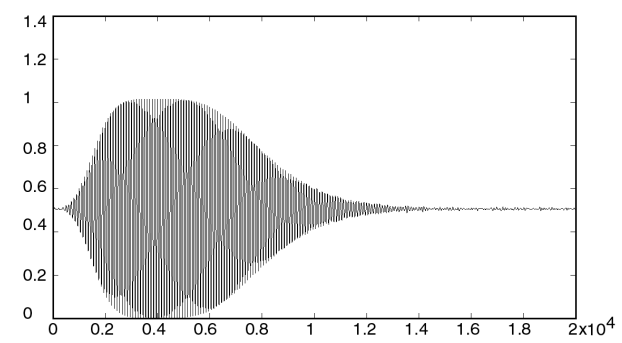

(c)

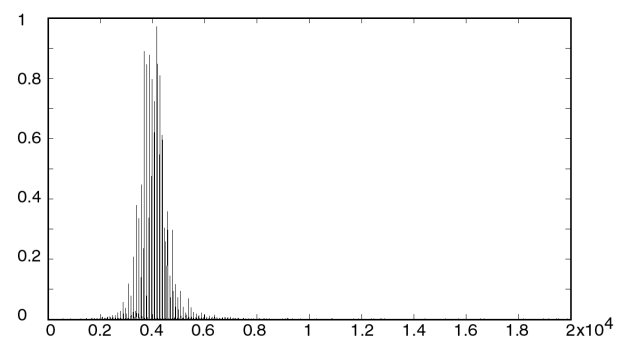

(b)

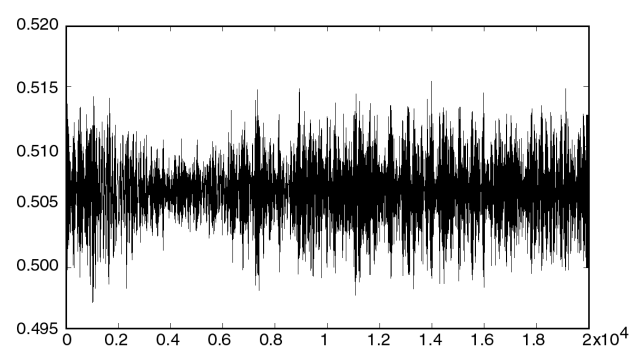

(d)

FIGURE 4. (a) Syntentic pulse. Frequency: $10 \mathrm{~Hz}, \alpha=4, \beta=1, \mathrm{~A}=w(t) / \max (w(t))$, signal length: 20 sec, samples per second: 1000, Gaussian noise: $2 \%$. (b) Rényi filtered signal. PWD window: $N=8$ samples, signal bias: +1 . (c) Fisher filter by correlating the signal with a sinusoidal wave of the same frequency as the input $(10 \mathrm{~Hz})$. (d) The same Fisher filter, but using a reference wave of $50 \mathrm{~Hz}$.

The next example is a synthetic pulse trains signal generated with the 'pulstran' Matlab function. This function generates a pulse train that is composed by the sum of multiple delayed interpolations of variations of the prototype pulse where samples are identically zero outside the pulse time intervals (see Fig. 5a). A window of $N=8$ data values has been preferred to calculate the PWD of signals around 300 samples per cycle. First, the signal has been analyzed through a classical wavelet decomposition scheme. Fig. 5b shows the wavelet coefficients for approximation at level 3 and for details at levels $3,2,1$. Fig. 5c shows the results of applying the current described 
method. From such figure, one can observe how precisely the extraction of the normalized Rényi entropy is able to delineate the signal's events of interest, in contrast with the wavelet-based method.

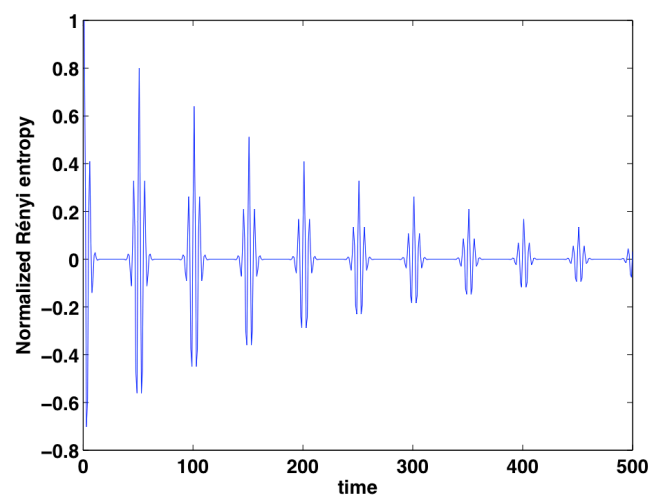

(a)

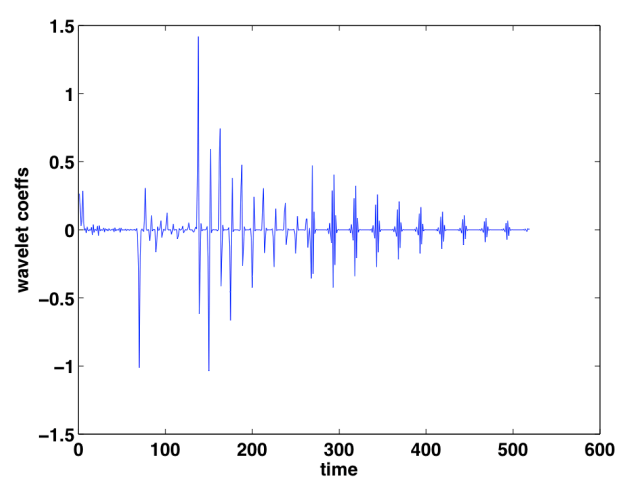

(b)

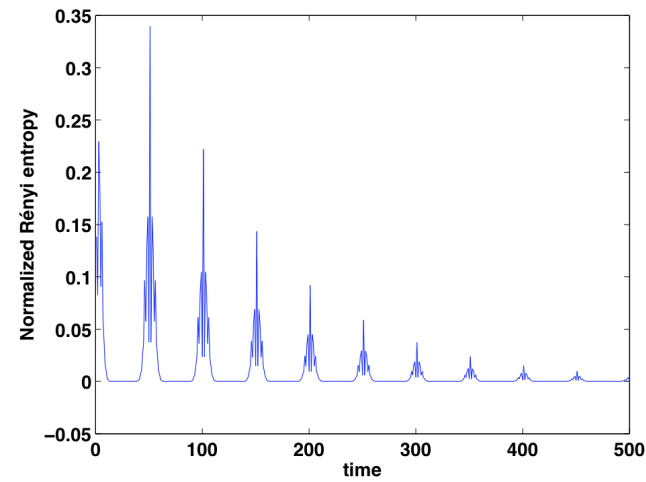

(c)

FIGURE 5. (a) Periodic Gaussian pulse signal at $10 \mathrm{~Hz}(50 \%$ bandwidth). The pulse frequency is $1 \mathrm{~Hz}$, sample rate is $50 \mathrm{~Hz}$ and pulse train length is $10 \mathrm{msec}$. The amplitude repetition is attenuating by 0.8 each time. (b) Wavelets coefficients using Daubechies 'db4' basis with 3 levels of decomposition ('wavedec' Matlab function). (c) Events detected by the Rényi-based method. The PWD has been calculated using a window of $N=8$ data. Signal bias: +1 .

\section{CONCLUSIONS}

A new method for events detection in seismic time series signals, based on a local Rényi entropy measure, has been described in this paper. The method has been tested with natural and synthetic seismic signals and the results have been compared with another classical event detection methods, such as Fisher and wavelet filtering. It presents some interesting properties, such as its localizing sharpness and high noise robustness. Examples shown that this method can provide a way of discriminating and identifying unsupervised events in discrete-time signals, that could be useful for facilitating the identification of events or even as input information to an automatic recognition system. Further research will focus on analyzing the influence of parameter estimation in the events detection and its evaluation. For example, position, and amplitude of identifiable events are expected to be determined in further 
supervised applications. The described approach could be of interest for the detection of more complicated events, e.g. precursor events in seismic time series or in epileptic seizures.

\section{ACKNOWLEDGMENTS}

This work has been partially supported by the following grants: TEC2007-67025; TEC2005-24739-E; TEC2005-24046-E; 2004CZ0009 from the Spanish Ministry of Science and Innovation.

\section{REFERENCES}

[1] Hagen, H.C., "The application of principal components analysis to seismic datasets", Geoexploration, 20, pp. 93-111, 1982

[2] Bois, P., "Autorregresive pattern recognition applied to the delimitation of oil and gas reservoirs", Geophysical Prospecting, 28, pp. 572-591, 1980

[3] Dumay, J., Fournier, F. "Multivariate statistical analyses applied to seismic facies recognition", Geophysics 53, pp. 1151-59, 1988

[4] Lendzionowski, V., Walden, A.T. and White, R.E., "Seismic character mapping over reservoir intervals", Geophy. Prospecting 38, pp. 951-969, 1990

[5] Walden, A.T., "Spatial clustering : using simple summaries of seismic data to find the edge of an oil-field", Applied Statistics 43, pp. 385-398, 1994

[6] Grubb, H.J. and Walden, A.T. "Characterizing seismic time series using the discrete wavelet transform", Geophy. Prosp., 45, pp. 183-205, 1997

[7] Endo, T.E. and Murray, T., "Real-time Seismic Amplitude Measurement (RSAM): a volcano monitoring and prediction tool", Bull. Volcanol., 53, pp. 533-545, 1991

[8] Bartosch, T. and Seidl, D. "Spectrogram análisis of selected tremor signals using short-time Fourier transform and continuos wavelet transform", Ann. Geofis, 42, 497-506, 1999

[9] Zou, R., Cupples, W.A., Yip, K.P., Holstein-Rathlou, N.H. and Chon, K.H. "Time-varying properties of renal autoregulatory mechanisms" IEEE Transactions on Biomedical Engineering, Vol. 49, No. 10, 2002

[10] Yadollahi, A., Moussavi, Z., Shamsollahi1, M.B. and Ahmadinejad, Z., "Heart sounds localization using lung sounds entropy”, Int. J. Sci. Res., Vol. 15 (2005), in press.

[11] Stankovic, L."A measure of some time-frequency distributions concentration", Signal Processing 81, pp. 621631,2001

[12] Shannon, C.E. and Weaver, W. The Mathematical Theory of Communication. The University of Illinois Press, Urbana, Chicago, London, 1949.

[13] Rényi, A. "Some fundamental questions of information theory”. In Pál Turán, editor, Selected Papers of Alfréd Rényi, volume 3, pp. 526-552. Akadémiai Kiadó, Budapest, 1976. (Originally: MTA III. Oszt. Közl., 10, 1960, pp. 251-282).

[14] Sang, T.H. and Williams, W.J. "Rényi information and signal dependent optimal kernel design". Proceedings of the ICASSP, vol. 2, pp. 997-1000, 1995

[15] Stankovic, L.J. “A method for time-frequency analysis”, IEEE Trans. Signal Process. 42 , pp.225-229, 1994

[16] Vakman, D. "Optimum signals which minimize partial volume under an ambiguity surface", Radio Eng. Electron. Phys. 23 , pp.1260-1268, 1968

[17] Williams, W.J., Brown, M.L. and Hero, A.O. "Uncertainity, information and time \} frequency distributions", SPIE Adv. Signal Process. Algebra Arch. Imp. 1566, pp. 144-156, 1991

[18] Flandrin, P., Baraniuk, R.G., Michel, O."Time-frequency complexity and information”, Proceedings of the ICASSP, vol. 3, pp. 329-332, 1994

[19] Pitton, J., Loughlin, P. and Atlas, L. "Positive time-frequency distributions via maximum entropy deconvolution of the evolutionary spectrum", Proc. ICASSP, vol. IV, pp. 436-439, 1993.

[20] Wigner, E. "On the Quantum Correction for Thermodynamic Equilibrium.” Physical Review, Vol. 40, pp. 749759,1932 
[21] Gabarda, S.; Cristóbal, G.; Martínez-Alajarín, J.; Ruiz, R. "Detection of Events in Biomedical Signals by a Rényi Entropy Measure", Information Optics: 5th International Workshop on Information Optics (WIO'06). AIP Conference Proceedings, Volume 860, pp. 210-219 (2006).

[22] Köhler, A., Ohrnberger, M., Riggelsen, C., Scherbaum, F. "Unsupervised Feature Selection for Pattern Search in Seismic Time Series", JMLR: Workshop and Conference Proceedings 4,106-121 (2008)

[23] Cohen, L. "Generalized phase-space distribution functions", J. Math. Physics, Vol. 7, pp. 781-786, 1966.

[24] Jacobson, L.D. and Wechsler, H. "Joint spatial/spatial-frequency representation", Signal processing, Vol. 14, 37-68, 1988.

[25] Claasen, T. A. C. M. and Mecklenbräuker, W. F. G. "The Wigner distribution - A Tool for Time Frequency Analysis,” Parts I-III." Philips J. Research, Vol. 35, 217-250, 276-300, 372-389, 1980.

[26] Brenner, K.H. "A discrete version of the Wigner distribution function", Proc. EURASIP, Signal Processing II: Theories and Applications, pp. 307-309, 1983

[27] Eisberg, R. \& Resnick, R. “Quantum Physics”, Wiley, 1974.

[28] Plešinger, A. and Kolár, P. "Interactive demonstration of the effect of causal and acausal frequency filtering on seismic signals", Geophysical Institute, Academy of Sciences of the Czech Republic, dsp.vscht.cz/konference_matlab/matlab02/kolar_p.pdf

[29] van den Hazel, G.J. "Seismic event detection using arrays - A comparison of 2 approaches", Bachelor Thesis, Utrecht University, 2007, www.knmi.nl/ evers/other-publications/bachelorthesisVdHazel.pdf

[30] Melton, B. and Bailey, L. "Multiple signal correlators", Geophysics, XXII(3), pp. 565-588 , 1957 